\title{
Image reconstruction using spectroscopic and hyperspectral information for compressive terahertz imaging
}

\author{
Zhimin Xu and Edmund Y. Lam* \\ Imaging Systems Laboratory, Department of Electrical and Electronic Engineering, The University of Hong Kong, \\ Pokfulam Road, Hong Kong, China \\ *Corresponding author: elam@eee.hku.hk
}

Received December 10, 2009; revised May 19, 2010; accepted May 25, 2010; posted May 25, 2010 (Doc. ID 121306); published June 16, 2010

Terahertz $(\mathrm{THz})$ time-domain imaging is an emerging modality and has attracted a lot of interest. However, existing $\mathrm{THz}$ imaging systems often require a long scan time and sophisticated system design. Recently, a new design incorporating compressed sensing (CS) leads to a lower detector cost and shorter scan time, in exchange for computation in an image reconstruction step. In this paper, we develop two reconstruction algorithms that can estimate the underlying scene as accurately as possible. First is a single-band CS reconstruction method, where we show that by making use of prior information about the phase and the correlation between the spatial distributions of the amplitude and phase, the reconstruction quality can be significantly improved over previously published methods. Second, we develop a method that uses the multi-frequency nature of the $\mathrm{THz}$ pulse. Through effective use of the spatial sparsity, spectroscopic phase information, and correlations across the hyperspectral bands, our method can further enhance the recovered image quality. This is demonstrated by computation on a set of experimental THz data captured in a single-pixel THz system. (C) $2010 \mathrm{Optical}$ Society of America

OCIS codes: $110.6795,100.3010,100.3020,100.3190,110.1758$.

\section{INTRODUCTION}

In recent years, advances in terahertz $(\mathrm{THz})$ science have attracted increasing attention in time-domain $\mathrm{THz}$ imaging for a very diverse array of applications. These various potential applications range from biology and medical sciences [1] and non-destructive evaluation [2] to quality control processes [3] and homeland security [4]. In contrast to the conventional optical modality that is based on relative intensity measurements only, one important advantage of $\mathrm{THz}$ time-domain imaging systems is that the transient electric field itself can be measured, from which we can determine the amplitude and phase of each spectral component that makes up the entire $\mathrm{THz}$ pulse. Thus, $\mathrm{THz}$ imaging systems provide opportunities for spectroscopic studies, which are not possible with many competing modalities. Unfortunately, most existing $\mathrm{THz}$ imaging systems suffer from slow acquisition because of their raster-scanning nature [2,5,6]. For example, using one of the fastest raster-scanning time-domain $\mathrm{THz}$ imaging system to date [6], we still need 6 min to scan a $100 \mathrm{~mm}^{2}$ area at $0.25 \mathrm{~mm}$ resolution (equivalently, a $400 \times 400$ pixel image). Although recent efforts by using more sophisticated imaging technologies, such as interferometric and tomographic approaches, have shown preliminary successes, raster-scanning is still a latent factor that limits the acquisition rate of such systems. The high complexity and hardware requirements are also major limitations to their practical applications.

To meet the requirements of practical, time-crucial applications, Chan et al. [7,8] propose two fast time-domain $\mathrm{THz}$ imaging schemes by applying the compressed sens- ing (CS) theory $[9,10]$. In [7], the imaging process is accelerated by randomly sampling only a subset of the Fourier amplitude measurements. However, signal acquisition in this system still requires the $\mathrm{THz}$ receiver to raster scan the focal plane. To replace this mechanical rasterscanning, they then present a proof-of-concept singlepixel $\mathrm{THz}$ imaging setup in [8]. The system schematic is illustrated in Fig. 1. Instead of collecting the pixels/ voxels, a single detection element is used to sample the concentrated $\mathrm{THz}$ beams, which are spatially modulated with a set of random patterns. According to the CS theory, much fewer samples than the total number of image pixels are needed to fully reconstruct an image, which thus implement fast compressed imaging. In exchange for high-speed signal acquisition, the challenge of implementing such a system comes in developing efficient sparsifying representations and the corresponding algorithms for signal recovery from the linear measurements. The CS theory suggests the conditions that need to be fulfilled to allow nearly perfect reconstruction with a much smaller number of measurements. However, in reality, these conditions are hard to satisfy fully. In addition, the signal obtained from time-domain $\mathrm{THz}$ imaging systems is the transient electric field. That is, the received signal is complex $[8,11]$. Several CS reconstruction algorithms have been proven effective in dealing with real-valued signals, but for complex-valued signals, challenges still exist. The authors in $[12,13]$ extend the considerations to the complex domain. However, aside from the $\ell_{1}$ norm regularizer on the complex data, they did not exploit the other a priori information. 


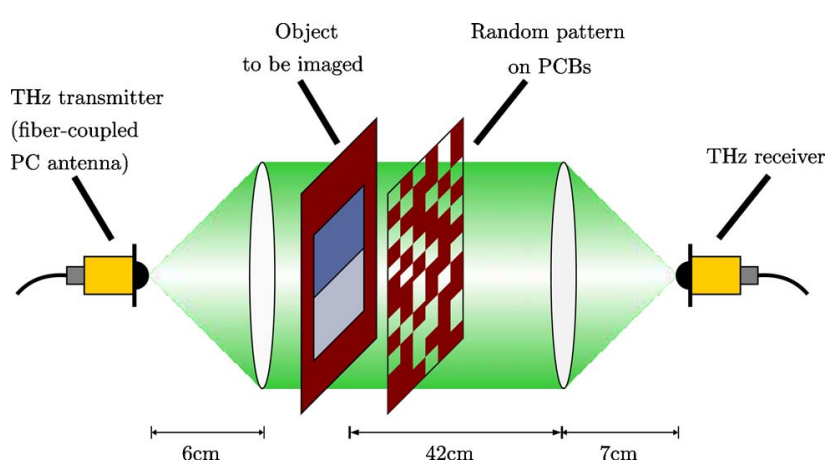

Fig. 1. (Color online) Schematic diagram of a single-pixel, pulsed $\mathrm{THz}$ imaging system based on that in [8].

In this paper, we present a reconstruction algorithm to estimate the underlying complex signals based on the single-pixel $\mathrm{THz}$ imaging system described in [8]. We show that by using the prior knowledge of the phase and properties contained in the system, our algorithm can significantly improve the reconstruction quality. In addition, we note that time-domain $\mathrm{THz}$ modalities, such as $\mathrm{THz}$ time-domain spectroscopy (THz-TDS) and $\mathrm{THz}$ imaging systems, also offer an important piece of information that is not commonly found in other ones: the $\mathrm{THz}$ pulse contains information at multiple frequencies, and therefore has hyperspectral information $[14,15]$. We thus consider further enhancing the reconstruction image quality by making use of such hyperspectral information. Preliminary work along this direction has been reported in [16].

This paper is organized as follows. Following a brief review in Subsection 2.A concerning the CS theory and the reconstruction techniques commonly used, we propose a sparse reconstruction method for signal recovery at a specific frequency in Subsection 2.B. The work presented here is an extension of our previous work in [17], and more technical details are provided. Then, in Subsection 2.C we introduce our partition-based multiscale hyperspectral image estimation approach for the complexvalued data. In Subsection 2.D, we combine the singleband sparse reconstruction method with multiscale hyperspectral image estimation and devise a hyperspectral image reconstruction algorithm for the compressive $\mathrm{THz}$ imaging system. For illustration purposes, we demonstrate in Section 3 the output of our algorithm on the practical $\mathrm{THz}$ data. Last, based on the experimental results, we draw our conclusions in Section 4.

\section{METHODS}

\section{A. Compressed Sensing Background}

Compressed sensing is concerned with encoding a sparse signal using a relatively small number of linear measurements and ensures accurate reconstruction with a very high probability when a sufficient condition called the restricted isometry property (RIP) is satisfied $[9,10]$. Mathematically, given an $M \times N$ measurement matrix $\Psi$ with $M<N$ and a vector $\mathbf{b}$ that denotes the linear measurements of an $N$-dimensional sparse signal $\mathbf{x}$, say $\mathbf{b}=\Psi \mathbf{x}$, the optimal estimate of the underlying sparse signal can be solved by

$$
\text { minimize }\|\mathbf{x}\|_{0} \quad \text { subject to } \Psi \mathbf{x}=\mathbf{b},
$$

with $\|\mathbf{x}\|_{0}$ the number of nonzero entries in $\mathbf{x}$, also called the $\ell_{0}$ norm. That is, we can estimate the underlying sparse/compressible signals (e.g., THz signals) by searching the sparsest solution satisfying $\Psi \mathbf{x}=\mathbf{b}$. But this is computationally intractable and highly sensitive to noise [18]. Consequently, an easier-to-solve linear program is used for searching the sparsest solution of $\Psi \mathbf{x}=\mathbf{b}$; that is,

$$
\text { minimize }\|\mathbf{x}\|_{1} \quad \text { subject to } \Psi \mathbf{x}=\mathbf{b} \text {, }
$$

where $\|\mathbf{x}\|_{1}=\sum_{i=1}^{N}\left|x_{i}\right|$ denotes the $\ell_{1}$ norm of a vector $\mathbf{x}$. In other words, one minimizes the $\ell_{1}$ norm of the signal instead of the sparsity itself.

To solve such an inverse problem, several methods and code packages have been developed, such as the SPGL1 algorithm [19,20], gradient projection for sparse reconstruction [21], $\ell_{1}$-magic [22], etc. Since the signal $\mathbf{x}$ considered in the conventional CS theory is real, most of the algorithms focus only on processing real-valued signals. Although some researchers have tried to extend their methods to the complex domain [19], the reconstruction quality in practical applications is often not satisfactory. For instance, one such attempt can be observed from Fig. 4 in [8]. If we consider the optimization problem in Eq. (2) carefully, we may note that for the complex case (i.e., $\mathbf{x}$ $\left.\in \mathcal{C}^{N}\right)$, the term $\|\mathbf{x}\|_{1}$ actually imposes constraints only on the amplitude of $\mathbf{x}$. So aside from sparsity of the amplitude intensity, any other a priori information (e.g., the phase) has not been exploited. The pulsed $\mathrm{THz}$ imaging systems are well-known for providing spectroscopic phase information, and this leads us to further considerations on the use of the phase. On the other hand, in the timedomain setups, such as the $\mathrm{THz}$ imaging systems in $[7,8]$, some underlying relations have not been used for further improvement in the image reconstruction. This includes the similarities between spatial intensity distributions of the amplitude and phase and the correlation across the hyperspectral bands. In the following sections, we demonstrate the capabilities of this prior knowledge in the recovered image quality improvement.

\section{B. Single-Band Compressed Sensing Reconstruction} The single-pixel pulsed $\mathrm{THz}$ imaging system in Fig. 1 implements a CS process. The basic principle behind such a CS imaging system can be described as, at a particular frequency $f_{k}$,

$$
\mathbf{b}\left(f_{k}\right)=\Psi \mathbf{x}\left(f_{k}\right)
$$

where $\mathbf{b}\left(f_{k}\right) \in \mathcal{C}^{M}$ is a column vector of measurements and $\mathbf{x}\left(f_{k}\right)$ represents the underlying $N \times N$ complex-valued signal ordered in an $N^{2} \times 1$ vector, spatially modulated by a set of random patterns that form the measurement matrix $\Psi \in \mathcal{R}^{M \times N^{2}}$. As mentioned above, the CS theory provides us with the mathematical basis for the accurate recovery of the original signals with only a few measurements (i.e., $M \ll N^{2}$ ). For simplicity, one could directly adapt the optimization program in the form of Eq. (2) to our case, just recognizing that the true and observed data are both complex. Since seeking the sparsest exact solution may be useless because of the additive noise, an ap- 
proximate reconstruction is often preferred. It then makes sense to replace Eq. (2) by

$$
\text { minimize }\left\|\mathbf{x}\left(f_{k}\right)\right\|_{1} \quad \text { subject to }\left\|\Psi \mathbf{x}\left(f_{k}\right)-\mathbf{b}\left(f_{k}\right)\right\|_{2} \leqslant \epsilon,
$$

with $\epsilon$ the tolerance to be defined. Unfortunately, such a simple extension to the complex domain can hardly produce a satisfactory estimation of the original scene. An example of this can be found in Fig. 4 in [8], and also in our experimental results in Section 3.

In our recent work [17], we propose an effective method that can significantly improve the reconstruction quality in combination with the phase information carried in the received $\mathrm{THz}$ pulses. The basic consideration relies on the fact that the phase should be smooth and not vary rapidly $[23,24]$. Hence, to interpret the smoothness constraint on the phase, we apply the similarities between the $i$ th pixel and the spatial neighborhood $\Omega_{i}$ of a certain size centered at pixel $i$. The size of $\Omega_{i}$ controls the degree of spatial smoothness. Meanwhile, this kind of smoothness constraint of the phase can also alleviate phase wrapping errors and noise to some degree. The size of the neighborhood area should be chosen according to the specific situation.

Let $\phi\left(x_{i}\right)$ and $\overline{\phi\left(\Omega_{i}\right)}$ be the phase value of the $i$ th pixel and the mean phase value in $\Omega_{i}$, respectively. The smoothness constraint on the phase intensities is then defined as

$$
\|\phi(\mathbf{x})-\overline{\phi(\mathbf{x})}\|_{2}=\left(\sum_{i=1}^{N^{2}}\left[\phi\left(x_{i}\right)-\overline{\phi\left(\Omega_{i}\right)}\right]^{2}\right)^{1 / 2} \leqslant \sigma,
$$

with

$$
\phi\left(x_{i}\right)=\left\{\begin{array}{ll}
-j \log \frac{x_{i}}{\left|x_{i}\right|}, & \text { if }\left|x_{i}\right| \geqslant T \\
0, & \text { otherwise }
\end{array} \in[-\pi, \pi) .\right.
$$

Here the parameter $\sigma$ is used to control the similarities between the $i$ th pixel and its nearest neighbors, and $T$ refers to a given threshold for separating the regions containing signal and noise only. Note that the reconstructed phase is arbitrary and carries no physical information at the location where the signal is very weak (i.e., the amplitude intensity is less than a given value $T$ ). Such artifacts will seriously affect the appearance of the restored results. We will show its effectiveness in detail in the experimental section.

In addition, many existing time-domain $\mathrm{THz}$ imaging systems, especially the one in Fig. 1, are based on a transmission-type spectroscopy. With no loss of generality, we assume that the object is piecewise homogeneous and has uniform thickness. Thus, we get one additional piece of prior knowledge about the original signals, which is that the smooth regions in the spatial distribution of amplitude should be the same as those in the spatial distribution of phase. Mathematically, let $x_{1}=A_{1} \exp \left\{j \phi_{1}\right\}$ and $x_{2}=A_{2} \exp \left\{j \phi_{2}\right\}$ be the complex intensities of two different pixels. If these two pixels are in the same homogeneous region, then $A_{1}=A_{2}$ and $\phi_{1}=\phi_{2}$. Accordingly, we can conclude that the first-order difference of the complex intensities in a homogeneous region is zero.
According to this assumption, if we define the total variation (TV) of a two-dimensional complex datum based on the $\ell_{0}$ norm as

$$
\sum_{i}\left\|\Delta_{i}^{h} \mathbf{x}\right\|_{0}+\left\|\Delta_{i}^{v} \mathbf{x}\right\|_{0}
$$

with $\Delta_{i}^{h}$ and $\Delta_{i}^{v}$ as linear operators corresponding to, respectively, horizontal and vertical first-order differences at pixel $i$, then minimizing the TV will be a more appropriate choice for sparse complex image reconstruction in $\mathrm{THz}$ systems. Such a definition is not only good for signal sparsifying, but also emphasizes the correlation between the spatial distributions of the amplitude and phase. That is, for the solution that produces the minimum value of that $\ell_{0}$ norm TV, any two different pixels in the same homogenous region will not only have the same amplitude intensities, but also the same phase. The other optimization processes, such as minimizing the $\ell_{1}$ norm of the signal itself or the coefficients in a certain transform domain, however, cannot efficiently utilize such spatial correlation. Meanwhile, although some transform operation (e.g., wavelet transform) may sparsify the signal, the spatial correlation between the amplitude and phase will be destroyed in that transform domain.

For tractable programming, we replace $\ell_{0}$ norm with $\ell_{1}$ norm, i.e.,

$$
\|\mathbf{x}\|_{\mathrm{TV}} \triangleq \sum_{i}\left|\Delta_{i}^{h} \mathbf{x}\right|+\left|\Delta_{i}^{v} \mathbf{x}\right|
$$

Such a form, which is defined as the complex-valued TV, also appears in [25], as holography is another common optical system that involves capturing complex signals $[26,27]$. Then minimizing Eq. (7) will give us the same solution as the one by minimizing the $\ell_{0}$ norm TV. At this point, we would like to mention that in some sources [28], another form of the $\ell_{1}$ norm TV on complex data has been used as follows:

$$
\|\mathbf{x}\|_{\mathrm{TV}} \triangleq \sum_{i}\left(\left|\Delta_{i}^{h} \mathbf{x}\right|^{2}+\left|\Delta_{i}^{v} \mathbf{x}\right|^{2}\right)^{1 / 2}
$$

However, the distinction between these two regularizers should be kept in mind, since, at least in our optimization problems, the definition in Eq. (7) leads to better results with much sharper edges, as illustrated in Section 3. Here and below, the term $\|\mathbf{x}\|_{\mathrm{TV}}$ refers to our first definition in Eq. (7).

Considering the case at a particular frequency $f_{k}$, the sparse reconstruction algorithm for the $\mathrm{CS} \mathrm{THz}$ imaging system can be interpreted as an optimization given by

$$
\begin{array}{ll}
\operatorname{minimize} & \left\|\mathbf{x}\left(f_{k}\right)\right\|_{\mathrm{TV}} \\
\text { subject to } & \left\|\Psi \mathbf{x}\left(f_{k}\right)-\mathbf{b}\left(f_{k}\right)\right\|_{2} \leqslant \epsilon, \\
& \left\|\phi\left(\mathbf{x}\left(f_{k}\right)\right)-\overline{\phi\left(\mathbf{x}\left(f_{k}\right)\right)}\right\|_{2} \leqslant \sigma,
\end{array}
$$

or, equivalently, by the following criterion

$$
\begin{aligned}
\hat{\mathbf{x}}\left(f_{k}\right)= & \underset{\mathbf{x}\left(f_{k}\right)}{\arg \min } \frac{1}{2}\left\|\Psi \mathbf{x}\left(f_{k}\right)-\mathbf{b}\left(f_{k}\right)\right\|_{2}^{2}+\lambda\left\|\mathbf{x}\left(f_{k}\right)\right\|_{\mathrm{TV}}+\mu \| \phi\left(\mathbf{x}\left(f_{k}\right)\right) \\
& -\overline{\phi\left(\mathbf{x}\left(f_{k}\right)\right)} \|_{2} .
\end{aligned}
$$


Such a solution of Eq. (10) can be found by convex programming methods, e.g., the nonlinear conjugate gradient method combined with backtracking line search.

\section{Complex Multiscale Hyperspectral Image Estimation}

Time-domain $\mathrm{THz}$ systems provide the capability to capture multiple spectral information in the far-infrared range. Recently, researchers have begun to pay more and more attention to $\mathrm{THz}$ hyperspectral imaging, such as security screening $[14,15]$. However, degradation (i.e., noise, blurring, etc.) of the observations acquired from hyperspectral imaging systems is always inevitable, and thus recovery of hyperspectral imagery from degraded observations becomes a vital task. A direct and naïve thought is to treat each spectral channel as an independent signal and then process them separately. The main shortcoming of such an approach is that it fails to account for the intercorrelated relationships present in the original hyperspectral signal. Researchers thus consider more sophisticated techniques to alleviate the degradation by taking not only the individual channels, but also the correlation across them, into account. One major approach is wavelet-based hyperspectral image estimation, because wavelet-based techniques can provide good spatial adaptivity by virtue of the well-localized property of wavelets in both time and frequency.

In [29,30], Atkinson et al. present a near-optimal hyperspectral image estimation scheme by addressing the different nature of spatial and spectral dimensions, which thus avoids the requirement of second-order signal statistics. In particular, based on some assumptions of the interchannel correlation, the hyperspectral signal can be approximately decorrelated in the spatial domain by using a 2-D discrete wavelet transform and the discrete Fourier transform (DFT) in the spectral domain. However, their work is essentially derived from the analysis of the hyperspectral Wiener filter, which is based on the assumption that the system is corrupted by additive white Gaussian noise. Besides, to choose a suitable wavelet basis to guarantee an optimal converge rate, some a priori information about the underlying signals has to be provided, e.g., the degrees of smoothness.

Another class of hyperspectral image estimation approaches, which can be categorized as partition-based methods, automatically adapts to the signal function without any user input or a priori notion. This enjoys the advantages of wavelet-based techniques, such as fast convergence rates. The work by Willett and Nowak [31] shows a way for Poisson intensity estimation of a singlechannel signal by using a penalized likelihood method on recursive dyadic partitions. Then Krishnamurthy and Willett [32] use such a partition-based multiscale Poisson intensity estimator in hyperspectral imaging reconstruction. While these methods are concerned with real-valued signal estimation from photon-limited observations corrupted by Poisson noise, in this paper we develop a partition-based multiscale estimation scheme for complex-valued hyperspectral signals gathered from time-domain $\mathrm{THz}$ imaging systems.

It is reasonable to think of a hyperspectral signal acquired from a time-domain $\mathrm{THz}$ imaging system as a three-dimensional dataset, where the first two dimensions correspond to the spatial locations and the third dimension indicates the index of each spectral band. Let $\mathbf{x}$ and $\mathbf{y}$ respectively denote the true complex hyperspectral signal and the distorted complex hyperspectral observation on a grid of size $N \times N \times K$, where $K$ represents the number of spectral bands. Our goal is to estimate $\mathbf{x}$ from $\mathbf{y}$ as accurately as possible. Note that in this subsection, the hyperspectral data $\mathbf{x}$ and $\mathbf{y}$ are of size $N \times N \times K$.

In accordance with [32], a key feature of the hyperspectral images is that the boundaries and singularities are located at the same spatial positions across all the spectral bands, no matter how bad the contrast or perceptibility is at some band. This is also true in our $\mathrm{THz}$ imaging scenario. In each spectral band, the underlying amplitude and phase intensities contain the same spatial boundaries in their respective domains. Therefore, in what follows we process the amplitude and phase separately. Specifically, we decompose the underlying signal $\mathbf{x}$ into the amplitude part $\mathbf{x}_{\mathbf{A}}$ and phase part $\mathbf{x}_{\phi}$, and equally define $\mathbf{y}_{\mathbf{A}}$ and $\mathbf{y}_{\phi}$ for the observation $\mathbf{y}$. Since the signals concerned here are basically spatially piecewise constant, to better adapt to the spatial distributions and preserve the discontinuities, we choose the multiscale approach by respectively performing recursive dyadic partitioning (RDP) in the amplitude and phase domains. The RDP process on an image produces a tree representation by recursively decomposing any part of an existing partition into dyadic squares, replacing a square by four similar squares of half the size [33]. Thus, the spatial RDPs can be represented in terms of quad-trees. Figure 2(a) gives a sample of an incomplete RDP and its tree. Since the partition defined by the RDP is not unique, we use penalized likelihood estimation to select the optimal partition $\hat{\mathcal{P}}$ that provides the best fit to the observations from the space of possible partitions $\Sigma_{\mathcal{P}}$. Each of the terminal squares of this dataadaptive RDP $\hat{\mathcal{P}}$ corresponds to a spatially homogeneous region. An example of such partitioning on a twodimensional image is shown in Fig. 2(b).

In particular, at the beginning, complete spatial RDPs in amplitude and phase domains are obtained by recursively partitioning their respective spaces into cells with dyadic side length until reaching the pixel-level resolution. Then starting from this finest level, we search backward to find the fittest partition over every level of the quad-tree representation by merging adjoining

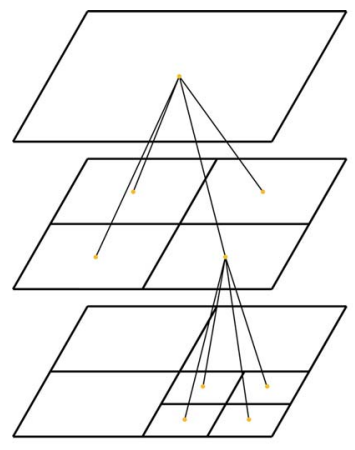

(a)

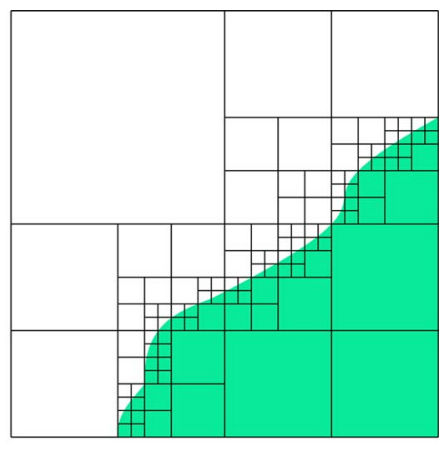

(b)
Fig. 2. (Color online) (a) A Example of an incomplete RDP and its quad-tree. (b) Sample partition of a two-dimensional image. 
squares based on the similarities of the intensities in the neighboring cells. We let $\mathcal{P}_{\mathbf{A}}$ and $\mathcal{P}_{\phi}$ denote the respective spatial RDP representations of amplitude and phase domains. Thus in each partition cell $c_{a} \in \mathcal{P}_{\mathbf{A}}$ and $c_{\phi} \in \mathcal{P}_{\phi}$, based on the respective likelihood of observing $\mathbf{y}_{\mathbf{A}}^{\left(c_{a}\right)}$ and $\mathbf{y}_{\phi}^{\left(c_{\phi}\right)}$ given the amplitude estimate $\mathbf{x}_{\mathbf{A}}^{\left(c_{a}\right)}$ and phase estimate $\mathbf{x}_{\phi}^{\left(c_{\phi}\right)}, p\left(\mathbf{y}_{\mathbf{A}}^{\left(c_{a}\right)} \mid \mathbf{x}_{\mathbf{A}}^{\left(c_{a}\right)}\right)$ and $p\left(\mathbf{y}_{\phi}^{\left(c_{\phi}\right)} \mid \mathbf{x}_{\phi}^{\left(c_{\phi}\right)}\right)$, we separately compute the following penalized likelihood (in a loglikelihood sense):

$$
\begin{aligned}
& \hat{\mathbf{x}}_{\mathbf{A}}^{\left(c_{a}\right)}=\underset{\mathbf{x}_{\mathbf{A}}^{\left(c_{a}\right)}}{\arg \min } L_{\mathbf{A}}^{\left(c_{a}\right)}=\underset{\mathbf{x}_{\mathbf{A}}^{\left(c_{a}\right)}}{\arg \min }\left\{-\log p\left(\mathbf{y}_{\mathbf{A}}^{\left(c_{a}\right)} \mid \mathbf{x}_{\mathbf{A}}^{\left(c_{a}\right)}\right)\right\}, \\
& \hat{\mathbf{x}}_{\phi}^{\left(c_{\phi}\right)}=\underset{\left.\mathbf{c}_{\phi}\right)}{\arg \min } L_{\phi}^{\left(c_{\phi}\right)}=\underset{\left(c_{\phi}\right)}{\arg \min }\left\{-\log p\left(\mathbf{y}_{\phi}^{\left(c_{\phi}\right)} \mid \mathbf{x}_{\phi}^{\left(c_{\phi}\right)}\right)\right\} .
\end{aligned}
$$

Apparently, the conditional probability functions $p\left(\mathbf{y}_{\mathbf{A}}^{\left(c_{a}\right)} \mid \mathbf{x}_{\mathbf{A}}^{\left(c_{a}\right)}\right)$ and $p\left(\mathbf{y}_{\phi}^{\left(c_{\phi}\right)} \mid \mathbf{x}_{\phi}^{\left(c_{\phi}\right)}\right)$ are directly related to the noise distributions in the particular $\mathrm{THz}$ system. While some work has been performed on modeling the distortions in the amplitude and phase domains [34], it is still an open problem in deciding an accurate noise estimation; in this paper, we choose the Gaussian distributions with mean zero and variance $\sigma_{\mathbf{A}}^{2}$ and $\sigma_{\phi}^{2}$ ) for the likelihood estimation, i.e.,

$$
\begin{aligned}
p\left(\mathbf{y}_{\mathbf{A}}^{\left(c_{a}\right)} \mid \mathbf{x}_{\mathbf{A}}^{\left(c_{a}\right)}\right)= & \left.\prod_{(i, j) \in c_{a}} \prod_{k=0}^{M-1} \frac{1}{\sqrt{2 \pi \sigma_{\mathbf{A}}{ }^{2}}}\right\}, \\
& \times \exp \left\{-\frac{\left[\mathbf{y}_{\mathbf{A}}(i, j, k)-\mathbf{x}_{\mathbf{A}}(i, j, k)\right]^{2}}{2 \sigma_{\mathbf{A}}{ }^{2}}\right\}, \\
p\left(\mathbf{y}_{\phi}^{\left(c_{\phi}\right)} \mid \mathbf{x}_{\phi}^{\left(c_{\phi}\right)}\right)= & \left.\prod_{(i, j) \in c_{\phi}} \prod_{k=0}^{M-1} \frac{1}{\sqrt{2 \pi \sigma_{\phi}{ }^{2}}}\right\} . \\
& \times \exp \left\{-\frac{\left[\mathbf{y}_{\phi}(i, j, k)-\mathbf{x}_{\phi}(i, j, k)\right]^{2}}{2 \sigma_{\phi}{ }^{2}}\right\} .
\end{aligned}
$$

Certainly, $p\left(\mathbf{y}_{\mathbf{A}}^{\left(c_{a}\right)} \mid \mathbf{x}_{\mathbf{A}}^{\left(c_{a}\right)}\right)$ and $p\left(\mathbf{y}_{\phi}^{\left(c_{\phi}\right)} \mid \mathbf{x}_{\phi}^{\left(c_{\phi}\right)}\right)$ can be modified for other kinds of distribution in the cases where the noise can be accurately estimated. Anyhow, such an algorithm gives the users high design freedom. Finally, the optimal RDP representations in the amplitude and phase domains are respectively calculated by selecting the ones with the minimal total penalized likelihood, and then the respective optimal estimators for the amplitude and phase intensities are the ones fit best to the observations over every cell in that optimal RDP, i.e.,

$$
\begin{aligned}
& \hat{\mathcal{P}}_{\mathbf{A}}=\underset{\mathcal{P}_{\mathbf{A}}}{\arg \min }\left\{\sum_{c_{a} \in \mathcal{P}_{\mathbf{A}}} L_{\mathbf{A}}^{\left(c_{a}\right)}+\eta_{\mathbf{A}}\left(\mathcal{P}_{\mathbf{A}}\right)\right\}, \\
& \hat{\mathbf{x}}_{\mathbf{A}}=\left\{\sum_{c_{a} \in \hat{\mathcal{P}}_{\mathbf{A}}} \hat{\mathbf{x}}_{\mathbf{A}}^{\left(c_{a}\right)}\right\},
\end{aligned}
$$

and

$$
\begin{aligned}
& \hat{\mathcal{P}}_{\phi}=\underset{\mathcal{P}_{\phi}}{\arg \min }\left\{\sum_{c_{\phi} \in \mathcal{P}_{\phi}} L_{\phi}^{\left(c_{\phi}\right)}+\eta_{\phi}\left(\mathcal{P}_{\phi}\right)\right\}, \\
& \hat{\mathbf{x}}_{\phi}=\left\{\sum_{c_{\phi} \in \hat{\mathcal{P}}_{\phi}} \hat{\mathbf{x}}_{\phi}^{\left(c_{\phi}\right)}\right\} \text {. }
\end{aligned}
$$

Note that in the above equations, the superscripts $c_{a}$ and $c_{\phi}$ restrict the areas of interest, and the variables with these superscripts are zero outside the corresponding areas. The terms $\eta_{\mathbf{A}}\left(\mathcal{P}_{\mathbf{A}}\right)$ and $\eta_{\phi}\left(\mathcal{P}_{\phi}\right)$ are penalties for controlling the spatial smoothness in the amplitude and phase domains, respectively. The values of these two penalties are respectively proportional to the number of cells in the partitions $\mathcal{P}_{\mathbf{A}}$ and $\mathcal{P}_{\phi}$. More technical details about the penalties can be found in [31]. Except for other hyperspectral image estimation methods, such as the ones mentioned in $[29,30]$, the key distinction is the use of the correlated relationships in the hyperspectral images. In our method, such relationships are exploited by separately forcing the spatial RDPs of the amplitude and phase dimensions at each spectral band to be the same.

\section{Compressed Sensing Hyperspectral Image Reconstruction}

Incorporating the single-band sparse reconstruction approach presented in Subsection 2.B with the complex multiscale hyperspectral image estimation technique in subsection 2.C, we devise a hyperspectral image reconstruction algorithm for the $\mathrm{CS} \mathrm{THz}$ imaging system. The algorithm flow chart is shown in Fig. 3. It consists of two alternating steps:

Step 1: Let $\hat{\mathbf{x}}^{(t)}$ be the input of the $t$ th iteration. Obtain the solution $\hat{\mathbf{y}}^{(t)}$ by computing Eq. (10) over each observed spectral band.

Step 2: Considering $\hat{\mathbf{y}}^{(t)}$ as the observations in this estimation process, perform our complex multiscale hyperspectral estimation method, then obtain $\hat{\mathbf{x}}^{(t+1)}$. Specifically, deal with the amplitude and phase parts separately and get the respective optimal estimators $\hat{\mathbf{x}}_{\mathbf{A}}^{(t+1)}$ and $\hat{\mathbf{x}}_{\phi}^{(t+1)}$ according to Eqs. (11)-(14). Combine the amplitude and phase and then obtain $\hat{\mathbf{x}}^{(t+1)}$.

These two steps are executed repeatedly, and the algorithm terminates when

$$
\frac{\left\|\hat{\mathbf{x}}^{(t+1)}-\hat{\mathbf{x}}^{(t)}\right\|_{1}}{\left\|\hat{\mathbf{x}}^{(t)}\right\|_{1}}
$$

is small enough.

\section{RESULTS AND DISCUSSION}

\section{A. Single-Band CS Reconstruction Results}

We expand on the preliminary results first presented in [17] with further experiments and analyses. The experimental data are acquired with the help of Wai Lam (William) Chan and Daniel M. Mittleman of Rice University, who are the designers of the single-pixel $\mathrm{THz}$ imaging system [8]. The test object is a rectangular hole in an opaque screen filled with two transparent plastic plates of different thickness, as shown in Fig. 4. Figure 5 shows the CS reconstruction results by using different approaches 


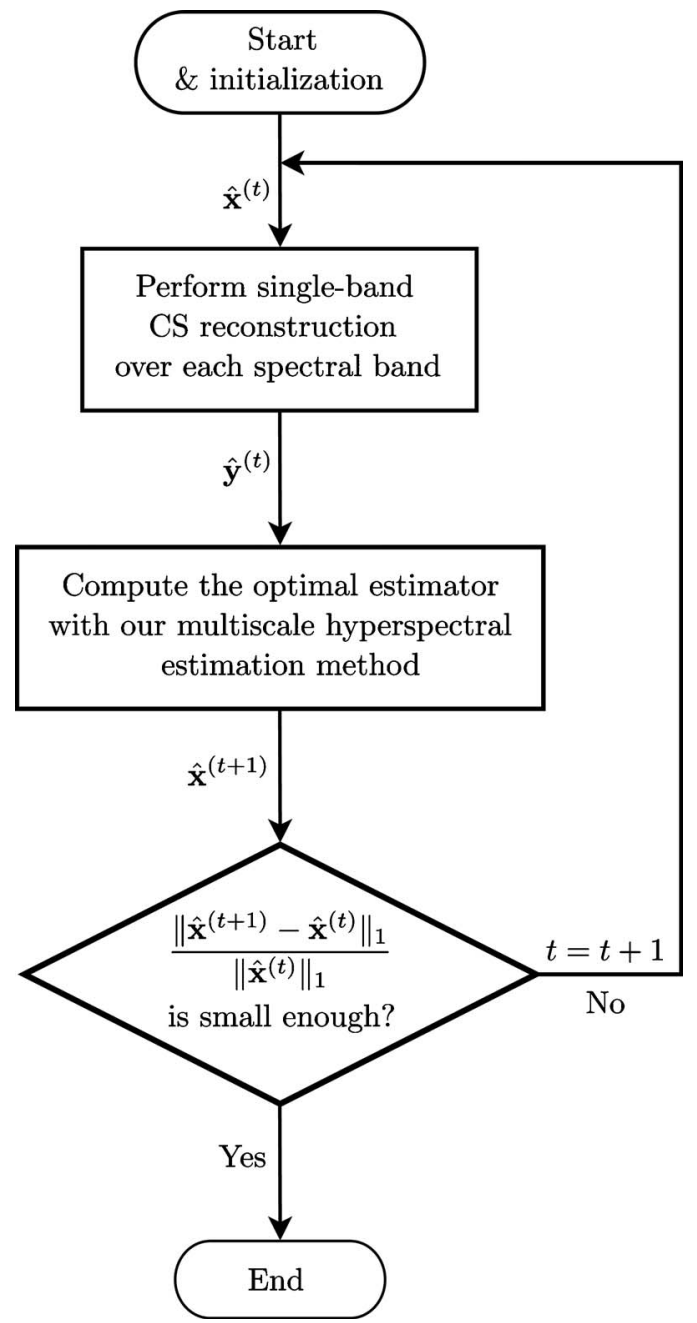

Fig. 3. Hyperspectral image reconstruction algorithm flow chart.

only from the linear measurements acquired at a specific frequency $(0.1 \mathrm{THz})$. In these experiments, 600 measurements are used to estimate the original complex signal of size $32 \times 32$.

We first perform the reconstruction by directly minimizing the $\ell_{1}$ norm of the underlying signal itself. The re-

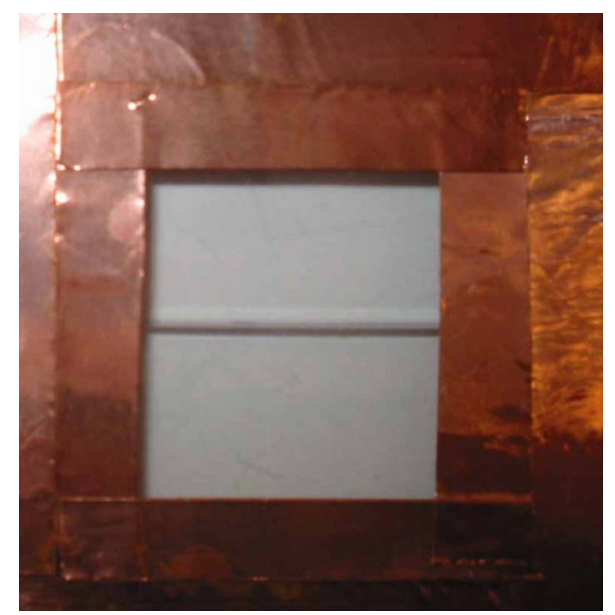

Fig. 4. (Color online) Rectangular object mask [17].

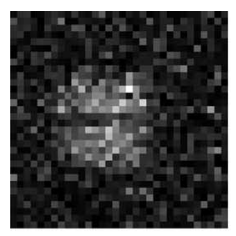

(a)

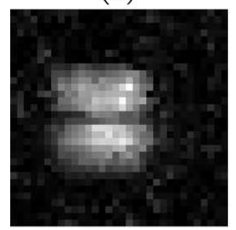

(c)

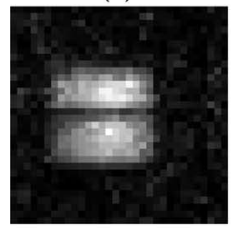

(e)

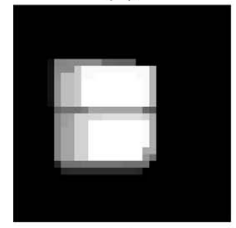

(g)

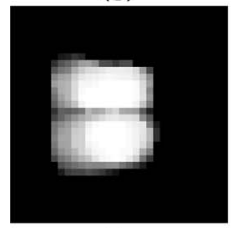

(i)

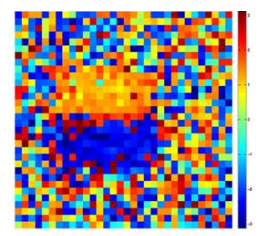

(b)

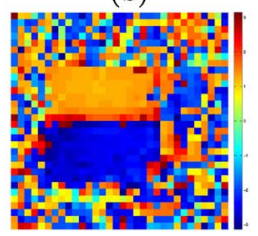

(d)

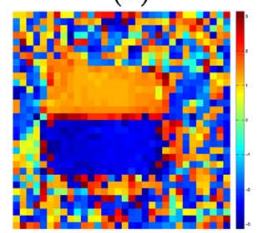

(f)

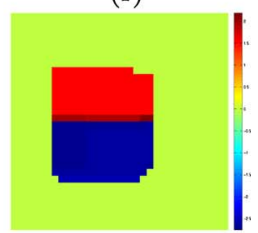

(h)

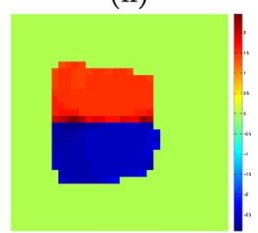

(j)
Fig. 5. (Color online) CS reconstruction results on the singleband $\mathrm{THz}$ data at the frequency of $0.1 \mathrm{THz}$. Each image is of size $32 \times 32$. The left column shows the amplitude images, and the phase images are in the right column. (a), (b) Results obtained by directly minimizing the $\ell_{1}$ norm of the underlying signal itself. (c), (d) Results reconstructed by minimizing the $\ell_{1}$ norm of the Daubechies-4 wavelet coefficients. (e), (f) Results obtained by using Daubechies-8 wavelet transform for sparsification. (g), (h) Results obtained by applying our single-band CS reconstruction method with Eq. (7) as the complex TV norm. (i), (j) Results obtained by using our single-band approach with the TV norm in the form of Eq. (8).

sults are shown in Figs. 5(a) and 5(b). Figures 5(c) and 5(d) and Figs. 5(e) and 5(f) are the respective results by using two different wavelet functions (i.e., Daubechies-4 wavelet and Daubechies-8 wavelet) for sparsification. These results are obtained by using the Matlab code package of SPGL1 algorithm [20]. The SPGL1 algorithm in [19] is an often used method which is designed to solve the conventional sparse reconstruction problem expressed in Eq. (4). Moreover, the SPGL1 algorithm is one of a few approaches which can deal with the problems in the complex domain. Although the improvement is recognizable in comparison with the direct $\ell_{1}$ norm minimization method, the visual quality of these recoveries is still unsatisfactory. For example, one still cannot identify the edges of each plastic plate, and the artifacts distributed in the background of both amplitude and phase images are quite obvious. 


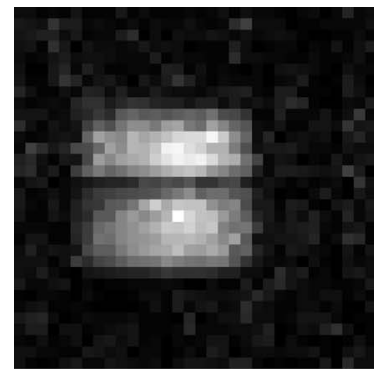

(a)

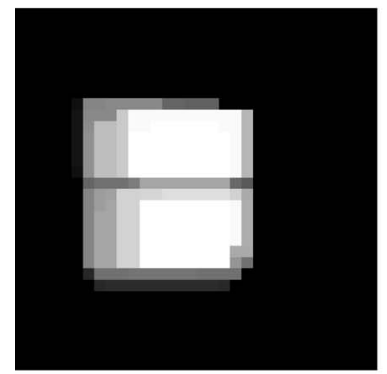

(c)

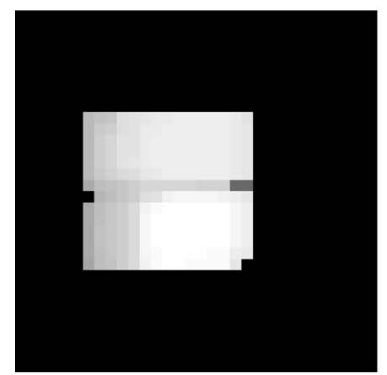

(e)

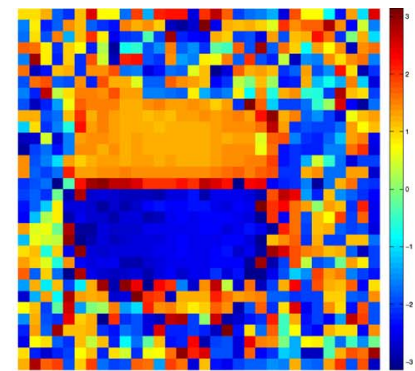

(b)

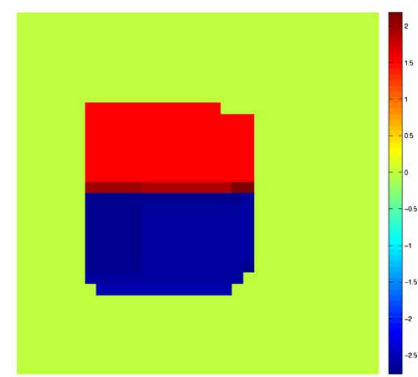

(d)

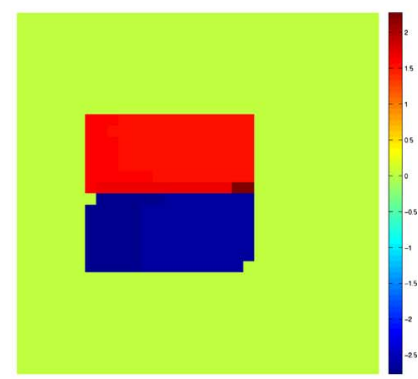

(f)
Fig. 6. (Color online) Hyperspectral reconstruction results with the practical $\mathrm{THz}$ data of size $600 \times 16$. (a), (b) Amplitude and phase obtained by minimizing the $\ell_{1}$ norm of the Daubechies- 8 wavelet coefficients only with the measurements at $0.1 \mathrm{THz}$. (c), (d) Amplitude and phase reconstructed by using our single-band reconstruction method only with the measurements at $0.1 \mathrm{THz}$ (e), (f) Amplitude and phase obtained by performing our proposed hyperspectral algorithm with data across all 16 spectral bands, displayed at $0.1 \mathrm{THz}$.

Next, we show the recovered amplitude and phase images by performing our single-band CS reconstruction methods. Note that in Subsection 2.B, we introduce two different forms of the complex TV norm, i.e., Eqs. (7) and (8). Figures 5(g) and 5(h) correspond to using Eq. (7), and the results with the TV norm of Eq. (8) are shown in Figs. $5(\mathrm{i})$ and $5(\mathrm{j})$. From the results, we can easily see that our single-band algorithm with either of these two forms of TV norm outperforms the conventional method. Yet the distinction between these two different forms also needs to be dealt with. The recovery with the form of Eq. (7) yields images consisting of smoother regions with much sharper edges. Hence, in the following experiments, we choose it as our complex TV regularizer. In addition, in the phase smoothing regularizer, the smoothness degree is controlled by the size of the neighborhood area around each pixel. Since too large a window will cause oversmoothing in the estimation, accordingly we use a window of $3 \times 3$ pixels.
B. CS Hyperspectral Multiscale Reconstruction Results In the experiments discussed below, we demonstrate the effectiveness of our proposed CS hyperspectral image reconstruction method on a set of practical hyperspectral $\mathrm{THz}$ measurements. The experimental platform is the same as in the previous experiment. Since each measurement obtained from the time-domain $\mathrm{THz}$ system is actually a whole pulse signal containing frequency information across the $\mathrm{THz}$ frequency range, we sample the measurements at 16 spectral bands uniformly distributed over the frequency range between $0.1 \mathrm{THz}$ and $0.2 \mathrm{THz}$. We first conduct the experiments with 600 measurements at each spectral band. Therefore, our goal is to find the optimal estimation of the underlying hyperspectral data of size $32 \times 32 \times 16$ with only $600 \times 16$ linear measurements.

Figures 6(a) and 6(b) show the amplitude and phase images obtained by applying the conventional CS reconstruction method with the information only at $0.1 \mathrm{THz}$.

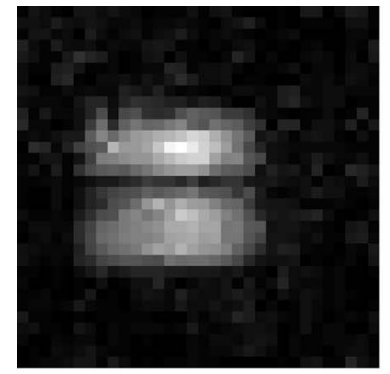

(a)

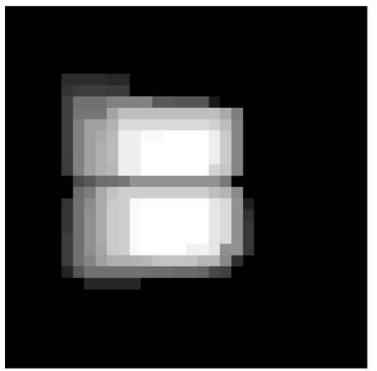

(c)

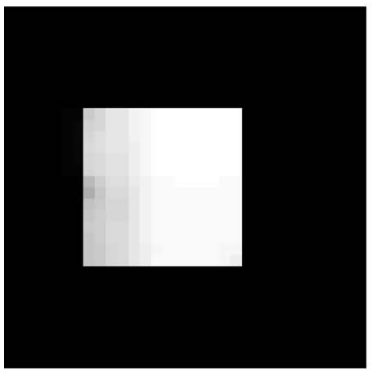

(e)

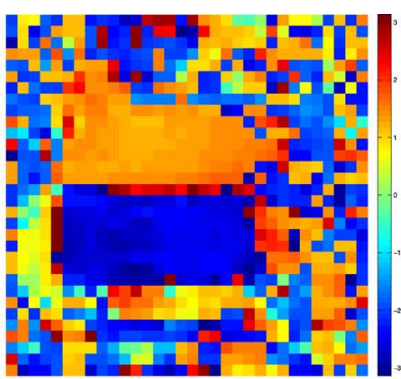

(b)

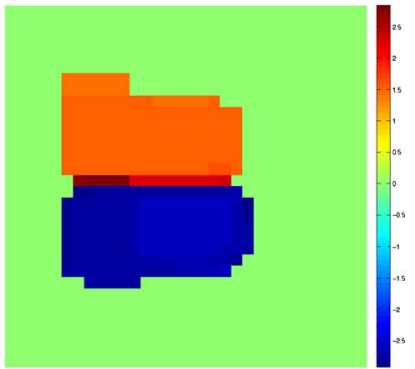

(d)

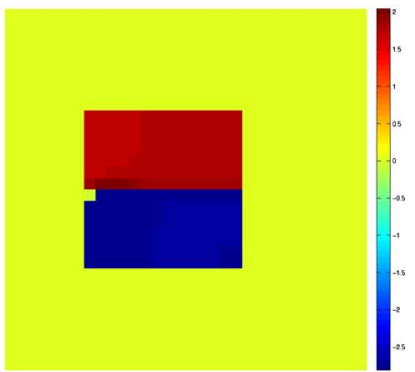

(f)
Fig. 7. (Color online) Hyperspectral reconstruction results with the practical $\mathrm{THz}$ data of size $400 \times 16$. (a),(b) Amplitude and phase obtained by minimizing the $\ell_{1}$ norm of the Daubechies- 8 wavelet coefficients only with the measurements at $0.1 \mathrm{THz}$. (c), (d) Amplitude and phase reconstructed by using our single-band reconstruction method only with the measurements at $0.1 \mathrm{THz}$. (e), (f) Amplitude and phase obtained by performing our proposed hyperspectral algorithm with data across all 16 spectral bands, displayed at $0.1 \mathrm{THz}$. 
The amplitude and phase reconstructed by using our single-band reconstruction algorithm in Subsection 2.B at the same frequency are shown in Figs. 6(c) and 6(d). In agreement with our previous discussion, the latter performs much better than the conventional method. However, these methods fail to preserve some fine edges, and we still cannot easily estimate the object edges accurately.

Now let us compare our proposed hyperspectral algorithm, shown in Figs. 6(e) and 6(f), with two reconstruction approaches that are based only on the consideration of single-band information. Visually, the reconstruction quality is significantly improved in both the amplitude and phase dimensions, i.e., clearer contours with less noticeable artifacts and smoother in the homogeneous regions. More importantly, the amplitude and phase recoveries corresponding to our proposed hyperspectral method are more coincident with the correlation between the amplitude and phase (see Subsection 2.B) and also closer to the reality that the two plastic plates are homogeneous. In addition, since each pixel corresponds to a $1 \mathrm{~mm}$ area, we get the size of the outer rectangular by counting pixels $(15 \mathrm{~mm} \times 14 \mathrm{~mm})$, which is equal to the actual measurement.

Furthermore, we also experiment with decreasing the number of the measurements to $400 \times 16$ (see Fig. 7). We can see that the results reconstructed from only the information at a single spectral band are still worse in quality, compared with our hyperspectral recovery. Our hyperspectral algorithm produces almost the same results, even though fewer measurements are used for reconstruction.

\section{CONCLUSION}

This paper studies methods for accurate image recovery in compressive $\mathrm{THz}$ imaging. First, we present a singleband CS reconstruction method. Unlike the conventional approach, which emphasizes the sparsity of only the amplitude, our single-band algorithm results in significant improvement by adding control on the phase and involving the correlation between the spatial distributions of amplitude and phase into the reconstruction process. Second, the hyperspectral nature of the $\mathrm{THz}$ pulse inspires us to devise a CS hyperspectral image reconstruction algorithm. In addition to the features of the single-band one, our hyperspectral method effectively employs correlations across the hyperspectral bands and shows good performance in preserving edges and alleviating artifacts in both amplitude and phase domains. Experimental results support these claims. Although the discussion in this paper is mainly based on the single-pixel $\mathrm{THz}$ imaging system in [8], many of contributions and discoveries can be readily utilized in other $\mathrm{THz}$ systems, such as time-domain $\mathrm{THz}$ spectroscopies.

\section{ACKNOWLEDGMENTS}

This work was supported in part by the Research Grants Council of the Hong Kong Special Administrative Region, China, under Projects HKU 713906 and 713408. We ac- knowledge the help of Wai Lam (William) Chan and Daniel M. Mittleman of Rice University in providing the experimental data.

\section{REFERENCES}

1. E. Pickwell and V. P. Wallace, "Biomedical applications of terahertz technology," J. Phys. D: Appl. Phys. 39, R301R310 (2006).

2. K. Kawase, Y. Ogawa, Y. Watanabe, and H. Inoue, "Nondestructive terahertz imaging of illicit drugs using spectral fingerprints," Opt. Express 11, 2549-2554 (2003).

3. S. Wietzke, C. Jöerdens, N. Krumbholz, B. Baudrit, M. Bastian, and M. Koch, "Terahertz imaging: a new nondestructive technique for the quality control of plastic weld joints,” J. Eur. Opt. Soc. Rapid Publ. 2, 07013 (2007).

4. R. M. Woodward, "Terahertz technology in global homeland security," Proc. SPIE 5781, 22-31 (2005).

5. N. Karpowicz, H. Zhong, C. Zhang, K.-I. Lin, J.-S. Hwang, J. Xu, and X.-C. Zhang, "Compact continuous-wave subterahertz system for inspection applications," Appl. Phys. Lett. 86, 054105 (2005).

6. D. Zimdars, "High speed terahertz reflection imaging," Proc. SPIE 5692, 255-259 (2005).

7. W. L. Chan, M. L. Moravec, R. G. Baraniuk, and D. M. Mittleman, "Terahertz imaging with compressed sensing and phase retrieval," Opt. Lett. 33, 974-976 (2008).

8. W. L. Chan, K. Charan, D. Takhar, K. F. Kelly, R. G. Baraniuk, and D. M. Mittleman, "A single-pixel terahertz imaging system based on compressed sensing," Appl. Phys. Lett. 93, 121105 (2008).

9. E. J. Candès, J. Romberg, and T. Tao, "Robust uncertainty principles: exact signal reconstruction from highly incomplete frequency information," IEEE Trans. Inf. Theory 52, 489-509 (2006).

10. D. L. Donoho, "Compressed sensing," IEEE Trans. Inf. Theory 52, 1289-1306 (2006).

11. W. L. Chan, J. Deibel, and D. M. Mittleman, "Imaging with terahertz radiation,” Rep. Prog. Phys. 70, 325-1379 (2007).

12. J. J. Fuchs, "Convergence of a sparse representations algorithm applicable to real or complex data," IEEE J. Sel. Top. Signal Process. 1, 598-605 (2007).

13. L. Chaâri, J.-C. Pesquet, A. Benazza-Benyahia, and P. Ciuciu, "Minimization of a sparsity promoting criterion for the recovery of complex-valued signals," presented at SPARS'09: Signal Processing with Adaptive Sparse Structured Representation, Saint Malo, France, 2009.

14. A. Luukanen, A. J. Miller, and E. N. Grossman, "Passive hyperspectral terahertz imagery for security screening using a cryogenic microbolometer," Proc. SPIE 5789, 127-134 (2005).

15. M. C. Kemp, A. Glauser, and C. Baker, "Recent developments in people screening using terahertz technology: seeing the world through terahertz eyes," Proc. SPIE 6212, 62120T (2006).

16. Z. Xu and E. Y. Lam, "Hyperspectral reconstruction in biomedical imaging using terahertz systems," in IEEE International Symposium on Circuits and Systems (IEEE, 2010), pp. 2079-2082.

17. Z. Xu, W. L. Chan, D. M. Mittleman, and E. Y. Lam, "Sparse reconstruction of complex signals in compressed sensing terahertz imaging," in Signal Recovery and Synthesis, OSA Technical Digest (CD) (Optical Society of America, 2009), paper STuA4.

18. E. J. Candès, M. B. Wakin, and S. P. Boyd, "Enhancing sparsity by reweighted $\ell_{1}$ minimization," J. Fourier Anal. Appl. 14, 877-905 (2008).

19. E. van den Berg and M. P. Friedlander, "Probing the pareto frontier for basis pursuit solutions," SIAM J. Sci. Comput. (USA) 31, 890-912 (2008).

20. E. van den Berg and M. P. Friedlander, "SPGL1: A solver for large-scale sparse reconstruction," http://www.cs.ubc.ca/ labs/scl/spgl1. 
21. M. A. T. Figueiredo, R. D. Nowak, and S. J. Wright, "Gradient projection for sparse reconstruction: Application to compressed sensing and other inverse problems," IEEE J. Sel. Top. Signal Process. 1, 586-597 (2007).

22. E. J. Candès and J. Romberg, " $\ell_{1}$-magic: Recovery of sparse signals via convex programming," http:// www.acm.caltech.edu/l1magic.

23. J. L. Marroquin, J. A. Quiroga, and R. Rodriguez-Vera, "Piecewise smooth phase reconstruction," Opt. Lett. 24, 1802-1804 (1999).

24. G. R. Brady, M. Guizar-Sicairos, and J. R. Fienup, "Optical wavefront measurement using phase retrieval with transverse translation diversity," Opt. Express 17, 624-639 (2009).

25. D. J. Brady, K. Choi, D. L. Marks, R. Horisaki, and S. Lim, "Compressive holography," Opt. Express 17, 13040-13049 (2009)

26. E. Y. Lam, X. Zhang, H. Vo, T.-C. Poon, and G. Indebetouw, "Three-dimensional microscopy and sectional image reconstruction using optical scanning holography," Appl. Opt. 48 H113-H119 (2009).

27. X. Zhang, E. Y. Lam, and T.-C. Poon, "Reconstruction of sectional images in holography using inverse imaging," Opt. Express 16, 17215-17226 (2008).

28. J. Ma and M. Fenn, "Combined complex ridgelet shrinkage and total variation minimization," SIAM J. Sci. Comput. (USA) 28, 984-1000 (2006).

29. I. Atkinson, F. Kamalabadi, and D. L. Jones, "Waveletbased hyperspectral image estimation," in IEEE International Geoscience and Remote Sensing Symposium IEEE, (2003), pp. 743-745.

30. I. Atkinson, F. Kamalabadi, S. Mohan, and D. L. Jones, "Asymptotically optimal blind estimation of multichannel images," IEEE Trans. Image Process. 15, 992-1007 (2006).

31. R. M. Willett and R. D. Nowak, "Multiscale poisson intensity and density estimation," IEEE Trans. Inf. Theory $\mathbf{5 3}$, 3171-3187 (2007).

32. K. Krishnamurthy and R. M. Willett, "Multiscale reconstruction of photon-limited hyperspectral data," in IEEE/SP 14th Workshop on Statistical Signal Processing, (IEEE, 2007), pp. 596-600.

33. D. L. Donoho and X. Huo, "Beamlets and multiscale image analysis," in Multiscale and Multiresolution Methods: Theory and Applications, T. J. Barth, T. Chan, and R. Haimes, eds., Lecture Notes in Computational Science and Engineering (Springer, 2001), pp. 149-196.

34. L. Duvillaret, F. Garet, and J.-L. Coutaz, "Influence of noise on the characterization of materials by terahertz timedomain spectroscopy,” J. Opt. Soc. Am. B 17, 452-461 (2000). 